ERHARD CRAMER (Oldenburg)

Udo KAMPS (Oldenburg)

MOHAMmad Z. RAQAB (Amman)

\title{
CHARACTERIZATIONS OF EXPONENTIAL DISTRIBUTIONS BY SPACINGS OF GENERALIZED ORDER STATISTICS
}

Abstract. Properties of spacings of generalized order statistics based on IFR and DFR distributions are shown to characterize exponential distributions.

1. Introduction. For order statistics from an exponential distribution with parameter $\lambda>0(\operatorname{Exp}(\lambda))$ it is well known that the normalized spacings are independent and $\operatorname{Exp}(\lambda)$-distributed. A variety of characterization results has been shown by means of related distributional properties. A detailed survey is given in Gather et al. (1998). In Kamps and Gather (1997), characterizations of exponential distributions with two spacings involved have been extended to using normalized spacings of generalized order statistics (cf. Kamps 1999) with a particular choice of the model parameters, i.e., $m_{1}=\ldots=m_{n-1}=m$, say. In the following we consider generalized order statistics with no restriction imposed on the parameters. Let $X(1, n, \widetilde{m}, k), \ldots, X(n, n, \widetilde{m}, k)$ be generalized order statistics based on the distribution function $F$ with parameters $\gamma_{1}, \ldots, \gamma_{n}>0$. For brevity, they are denoted by $X(1), \ldots, X(n)$. If $F \equiv \operatorname{Exp}(\lambda)$, then the normalized spacings

$$
D(1)=\gamma_{1} X(1), \quad D(r)=\gamma_{r}(X(r)-X(r-1)), \quad 2 \leq r \leq n,
$$

of generalized order statistics are also iid $\operatorname{Exp}(\lambda)$-distributed random variables (cf. Kamps 1995, p. 81).

Instead of requiring identical distributions of certain spacings we utilize two types of equations as characterizing properties given that the underlying

2000 Mathematics Subject Classification: Primary 62G30; Secondary 62E10.

Key words and phrases: order statistics, generalized order statistics, exponential distribution, hazard rate, characterizations. 
distributions have the IFR or DFR property. Namely, we make use of the identities $h_{D(r)}(0)=h_{D(s)}(0)$ for one pair $(r, s), 1 \leq r<s \leq n$, where $h=f /(1-F)$ denotes the hazard rate of $F$, and $E D(r)=E D(r+1)$ for one $r \in\{1, \ldots, n-1\}$, respectively.

2. Preliminaries. In Cramer and Kamps (2003) it is shown that generalized order statistics based on some distribution function $F$ can alternatively be defined by

$$
X(r)=F^{-1}\left(1-\prod_{j=1}^{r} B_{j}\right), \quad 1 \leq r \leq n,
$$

where $B_{1}, \ldots, B_{n}$ are independent power-function-distributed random variables with $P\left(B_{j} \leq x\right)=x^{\gamma_{j}}, x \in(0,1), 1 \leq j \leq n$. If $F$ is supposed to be absolutely continuous with density function $f$ then $X(r)$ has the density function

$$
f^{X(r)}(t)=\left(\prod_{j=1}^{r} \gamma_{j}\right) G_{r}\left(\bar{F}(t) \mid \gamma_{1}, \ldots, \gamma_{r}\right) f(t), \quad t \in \mathbb{R}
$$

where $G_{r}\left(\cdot \mid \gamma_{1}, \ldots, \gamma_{r}\right) \equiv G_{r, r}^{r, 0}\left(\cdot \mid \begin{array}{c}\gamma_{1}, \ldots, \gamma_{r} \\ \gamma_{1}-1, \ldots, \gamma_{r}-1\end{array}\right)$ denotes a particular Meijer's $G$-function (cf. Mathai 1993), and $\bar{F}=1-F$. The function $G_{r}\left(\cdot \mid \gamma_{1}, \ldots, \gamma_{r}\right)$ has some interesting properties. For instance, it is continuous on the interval $(0,1]$ with $G_{r}\left(1 \mid \gamma_{1}, \ldots, \gamma_{r}\right)=0$ (see, e.g., Cramer et al. 2003).

Subsequently, we need the following formulas for which we refer to Mathai (1993) and Cramer et al. (2003) $(t \in(0,1))$ :

$$
\begin{aligned}
G_{1}\left(t \mid \gamma_{1}\right)= & t^{\gamma_{1}-1} \\
t^{\alpha} G_{r}\left(t \mid \gamma_{1}, \ldots, \gamma_{r}\right)= & G_{r}\left(t \mid \gamma_{1}+\alpha, \ldots, \gamma_{r}+\alpha\right), \quad \alpha \in \mathbb{R}, \\
\frac{d}{d t} G_{r}\left(t \mid \gamma_{1}, \ldots, \gamma_{r}\right)= & \frac{1}{t}\left[\left(\gamma_{r}-1\right) G_{r}\left(t \mid \gamma_{1}, \ldots, \gamma_{r}\right)\right. \\
& \left.-G_{r-1}\left(t \mid \gamma_{1}, \ldots, \gamma_{r-1}\right)\right], \quad r \geq 2 .
\end{aligned}
$$

The density of the spacing $D(r), r \geq 2$, is given by

$$
\begin{aligned}
f^{D(r)}(y)= & \left(\prod_{j=1}^{r-1} \gamma_{j}\right) \int_{-\infty}^{\infty}\left(\frac{\bar{F}\left(y / \gamma_{r}+x\right)}{\bar{F}(x)}\right)^{\gamma_{r}-1} \\
& \times G_{r-1}\left(\bar{F}(x) \mid \gamma_{1}, \ldots, \gamma_{r-1}\right) h(x) f\left(y / \gamma_{r}+x\right) d x, \quad y>0,
\end{aligned}
$$

since

$$
\begin{aligned}
F^{X(r-1), X(r)}(x, t) & =P(X(r-1) \leq x, X(r) \leq t) \\
& =P\left(\prod_{j=1}^{r-1} B_{j}>\bar{F}(x), \prod_{j=1}^{r} B_{j}>\bar{F}(t)\right)
\end{aligned}
$$




$$
\begin{aligned}
& =\int_{-\infty}^{\infty} P\left(\prod_{j=1}^{r-1} B_{j}>\bar{F}(x), \prod_{j=1}^{r} B_{j}>\bar{F}(t) \mid \prod_{j=1}^{r-1} B_{j}=z\right) d P^{\prod_{j=1}^{r-1} B_{j}}(z) \\
& =\left(\prod_{j=1}^{r-1} \gamma_{j}\right) \int_{\bar{F}(x)}^{1} P\left(B_{r}>\bar{F}(t) / z\right) G_{r-1}\left(z \mid \gamma_{1}, \ldots, \gamma_{r-1}\right) d z
\end{aligned}
$$

and

$$
\begin{aligned}
& \frac{d}{d t} \frac{d}{d x} F^{X(r-1), X(r)}(x, t) \\
& =\left(\prod_{j=1}^{r} \gamma_{j}\right)\left(\frac{\bar{F}(t)}{\bar{F}(x)}\right)^{\gamma_{r}-1} G_{r-1}\left(\bar{F}(x) \mid \gamma_{1}, \ldots, \gamma_{r-1}\right) h(x) f(t) .
\end{aligned}
$$

In the proof of Theorem 3.1, the monotonicity of a ratio $\frac{G_{r}\left(1-\cdot \mid \gamma_{1}, \ldots, \gamma_{r}\right)}{G_{s}\left(1-\cdot \mid \gamma_{1}, \ldots, \gamma_{s}\right)}$ of $G$ functions is utilized. This property is of independent interest since it relates order statistics in the likelihood ratio ordering. Random variables $X$ and $Y$ with respective densities $f$ and $g$ are called (partially) ordered in the sense of likelihood ratio, $X \stackrel{\operatorname{lr}}{\leq} Y$, if $f / g$ is a decreasing function on the union of the supports of $X$ and $Y$ (cf. Shaked and Shanthikumar 1994, pp. 27-28).

The following lemma extends the corresponding assertion for order statistics (cf. Chan et al. 1991, Boland et al. 1998, p. 95) and record values (cf. Kochar 1990) to generalized order statistics.

LEMMA 2.1. Let $X(1), \ldots, X(n)$ be generalized order statistics based on a continuous distribution function $F$. Then

$$
X(r) \stackrel{\operatorname{lr}}{\leq} X(s), \quad 1 \leq r<s \leq n
$$

Proof. Let $B_{1}, \ldots, B_{n}$ be independent random variables with $P\left(B_{j} \leq x\right)$ $=x^{\gamma_{j}}, x \in(0,1), 1 \leq j \leq n$. Since the densities of $-\log B_{1}, \ldots,-\log B_{n}$ are logconcave, the application of Theorems 1.C.5 and 1.C.4 of Shaked and Shanthikumar (1994, pp. 30-31) together with (1) yields

$$
-\log \prod_{i=1}^{r} B_{i}=\sum_{i=1}^{r}-\log B_{i} \leq \sum_{j=1}^{\operatorname{lr}}-\log B_{j}=-\log \prod_{j=1}^{s} B_{j}
$$

and thus

$$
X(r)=F^{-1}\left(1-\prod_{i=1}^{r} B_{i}\right) \stackrel{\mathrm{lr}}{\leq} F^{-1}\left(1-\prod_{j=1}^{s} B_{j}\right)=X(s) .
$$

3. Characterization results. By assuming that generalized order statistics are based on an IFR or DFR distribution with some additional properties, exponential distributions can be characterized by the equation $h_{D(r)}(0)$ 
$=h_{D(s)}(0)$. This extends results of Gajek and Gather (1989) for order statistics and record values and of Kamps and Gather (1997) for generalized order statistics with the particular choice $m_{1}=\ldots=m_{n-1}=m$, say, of the model parameters, i.e., $\gamma_{j}=k+(n-j)(m+1), 1 \leq j \leq n, k>0$. Other characterizations of exponential distributions via two spacings of order statistics were derived by Ahsanullah $(1978,1981 \mathrm{a})$.

Theorem 3.1. Let $X(1), \ldots, X(n)$ be generalized order statistics based on $F$ with $F^{-1}(0+)=0, F$ strictly increasing on $(0, \infty)$, and with density function $f$ and hazard rate $h$ which are both continuous from the right. Moreover, let $F$ be IFR or DFR. Then $F \equiv \operatorname{Exp}(\lambda)$ for some $\lambda>0$ iff there exists a pair $(r, s), 1 \leq r<s \leq n$, such that the limits $h_{D(j)}(0)=$ $\lim _{x \rightarrow 0+} h_{D(j)}(x)$ are finite for $j \in\{r, s\}$ and

$$
h_{D(r)}(0)=h_{D(s)}(0) \text {. }
$$

Proof. Let $s>r \geq 2$. By using representation (6) and $F(0)=0$ we find

$$
h_{D(r)}(0)=\left(\prod_{j=1}^{r-1} \gamma_{j}\right) \int_{0}^{\infty} G_{r-1}\left(\bar{F}(x) \mid \gamma_{1}, \ldots, \gamma_{r-1}\right) h(x) f(x) d x,
$$

and, due to (2),

$$
\int_{0}^{\infty} G_{r-1}\left(\bar{F}(x) \mid \gamma_{1}, \ldots, \gamma_{r-1}\right) f(x) d x=\left(\prod_{j=1}^{r-1} \gamma_{j}\right)^{-1} .
$$

Hence,

$$
\begin{array}{r}
h_{D(r)}(0)=h_{D(s)}(0) \\
\Leftrightarrow\left(\int_{0}^{\infty} G_{r-1}\left(\bar{F}(x) \mid \gamma_{1}, \ldots, \gamma_{r-1}\right) h(x) f(x) d x\right) \\
\times\left(\int_{0}^{\infty} G_{r-1}\left(\bar{F}(x) \mid \gamma_{1}, \ldots, \gamma_{r-1}\right) f(x) d x\right)^{-1} \\
=\left(\int_{0}^{\infty} G_{s-1}\left(\bar{F}(x) \mid \gamma_{1}, \ldots, \gamma_{s-1}\right) h(x) f(x) d x\right) \\
\quad \times\left(\int_{0}^{\infty} G_{s-1}\left(\bar{F}(x) \mid \gamma_{1}, \ldots, \gamma_{s-1}\right) f(x) d x\right)^{-1} \\
\Leftrightarrow \int_{x \leq y}\left[\begin{array}{r}
{\left[G_{r-1}\left(\bar{F}(x) \mid \gamma_{1}, \ldots, \gamma_{r-1}\right) G_{s-1}\left(\bar{F}(y) \mid \gamma_{1}, \ldots, \gamma_{s-1}\right)\right.} \\
\left.-G_{s-1}\left(\bar{F}(x) \mid \gamma_{1}, \ldots, \gamma_{s-1}\right) G_{r-1}\left(\bar{F}(y) \mid \gamma_{1}, \ldots, \gamma_{r-1}\right)\right]
\end{array}\right. \\
\times \delta(x, y) \\
\times(h(x)-h(y)) f(x) f(y) d x d y=0
\end{array}
$$


(cf. Kamps and Gather 1997). Because of Lemma 2.1, the expression $\delta(x, y)$ is nonnegative for $x \leq y$. It is important to mention that the ratio

$$
\frac{G_{r-1}\left(1-\cdot \mid \gamma_{1}, \ldots, \gamma_{r-1}\right)}{G_{s-1}\left(1-\cdot \mid \gamma_{1}, \ldots, \gamma_{s-1}\right)}
$$

is strictly decreasing in $(0,1)$. This can be seen directly from the explicit representation of the $G$-functions in terms of (linearly independent) functions

i.e.,

$$
t^{\gamma_{j}-1}(-\log t)^{k_{v j}}
$$

$$
G_{r}\left(t \mid \gamma_{1}, \ldots, \gamma_{r}\right)=\sum_{v=1}^{l} \sum_{j=0}^{d_{v}-1} \frac{K_{v j}}{\left(d_{v}-1-j\right) ! j !} t^{\delta_{v}-1}(-\log t)^{d_{v}-j-1}
$$

where $K_{v 0}=\prod_{q=1, q \neq v}^{l}\left(\delta_{q}-\delta_{v}\right)^{-d_{q}}$,

$$
K_{v j}=\sum_{p=0}^{j-1} \sum_{q=1, q \neq v}^{l}(-1)^{p+1}\left(\begin{array}{c}
j-1 \\
p
\end{array}\right) \frac{p ! d_{q}}{\left(\delta_{q}-\delta_{v}\right)^{p+1}} K_{v, j-1-p}, \quad j \geq 1,
$$

and the following notations are used:

$$
\begin{aligned}
\gamma_{1}=\ldots=\gamma_{d_{1}}<\gamma_{d_{1}+1}=\ldots=\gamma_{d_{1}+d_{2}}<\ldots \\
\\
\quad \ldots<\gamma_{d_{1}+\ldots+d_{l-1}+1}=\ldots=\gamma_{d_{1}+\ldots+d_{l}}
\end{aligned}
$$

with $l \in\{1, \ldots, r\}$ and $\delta_{j}=\gamma_{d_{1}+\ldots+d_{j}}, j=1, \ldots, l$. Hence, $\delta_{1}<\ldots<\delta_{l}$ and $d_{j}$ denotes the multiplicity of $\delta_{j}$ in the sequence $\left(\gamma_{1}, \ldots, \gamma_{r}\right), j=1, \ldots, l$ (cf. Cramer and Kamps 2003).

A constant ratio $G_{r-1}\left(1-\cdot \mid \gamma_{1}, \ldots, \gamma_{r-1}\right) / G_{s-1}\left(1-\cdot \mid \gamma_{1}, \ldots, \gamma_{s-1}\right)$ with value $c$ on an interval $(\alpha, \beta) \subset(0,1), \alpha<\beta$, would lead to $c=1$ and $r=s$, which means that both functions coincide. Hence, $\delta(x, y)>0$ for $x<y$. Since $h$ is increasing or decreasing, we have $h(x)=h(y)$ for all $x<y$, i.e., a constant failure rate. Thus, the assertion is proved.

In the case $r=1$, we have

$$
f^{D(1)}(y)=\bar{F}^{\gamma_{1}-1}\left(y / \gamma_{1}\right) f\left(y / \gamma_{1}\right), \text { and hence } h_{D(1)}(0)=f(0) .
$$

Thus,

$$
\begin{aligned}
h_{D(1)}(0)=h_{D(s)}(0) & \\
\Leftrightarrow & f(0)=\left(\int_{0}^{\infty} G_{s-1}\left(\bar{F}(x) \mid \gamma_{1}, \ldots, \gamma_{s-1}\right) h(x) f(x) d x\right) \\
& \times\left(\int_{0}^{\infty} G_{s-1}\left(\bar{F}(x) \mid \gamma_{1}, \ldots, \gamma_{s-1}\right) f(x) d x\right)^{-1} \\
& \Rightarrow \int_{0}^{\infty} G_{s-1}\left(\bar{F}(x) \mid \gamma_{1}, \ldots, \gamma_{s-1}\right) f(x)(h(x)-f(0)) d x=0
\end{aligned}
$$


Since

$$
h(x) \stackrel{(\leq)}{\geq} h(0)=f(0), \quad x>0,
$$

the assertion follows for $r=1$ as well.

Obviously, the property $h_{D(s)}(0)=h(0)$ for some $2 \leq s \leq n$ characterizes exponential distributions (cf. Remark 2.1 in Gajek and Gather 1989 and Ahsanullah 1981b,a for order statistics and record values). From the case $r=1$ in the proof of Theorem 3.1, it is clear that the IFR or DFR assumption can be replaced by requiring that zero is an extremal point of the hazard rate $h$.

A related result for record values with random index has recently been shown by Iwińska (2001).

In Theorem 3.2, the equality of expected successive normalized spacings is used as a characterizing property. It is an extension of results of Ahsanullah (1981a,b) for order statistics and record values, and of Kamps and Gather (1997) for a particular subclass of generalized order statistics.

Theorem 3.2. Let $X(1), \ldots, X(n)$ be generalized order statistics based on $F$ and parameters $\gamma_{1} \geq \ldots \geq \gamma_{n}>0, F^{-1}(0+)=0, F(x)<1$ for all $x>0$, and let $F$ have the IFR or DFR property. Then $F \equiv \operatorname{Exp}(\lambda)$ for some $\lambda>0$ iff there exists $r, 1 \leq r \leq n-1$, such that $E D(r)=E D(r+1)$.

Proof. Let $r \geq 2$ and $F$ be IFR. By using (4)-(6), and Fubini's lemma twice we obtain

$$
\begin{aligned}
1-F^{D(r)} & (x)=\int_{x}^{\infty} f^{D(r)}(y) d y \\
\stackrel{(6)}{=} & \left(\prod_{j=1}^{r-1} \gamma_{j}\right) \int_{x}^{\infty} \int_{-\infty}^{\infty}\left(\frac{\bar{F}\left(y / \gamma_{r}+z\right)}{\bar{F}(z)}\right)^{\gamma_{r}-1} \\
& \times G_{r-1}\left(\bar{F}(z) \mid \gamma_{1}, \ldots, \gamma_{r-1}\right) h(z) f\left(y / \gamma_{r}+z\right) d z d y \\
= & \left(\prod_{j=1}^{r-1} \gamma_{j}\right) \int_{-\infty}^{\infty} \bar{F}^{-\gamma_{r}}(z) G_{r-1}\left(\bar{F}(z) \mid \gamma_{1}, \ldots, \gamma_{r-1}\right) f(z) \\
& \times \int_{x}^{\infty} \bar{F}^{\gamma_{r}-1}\left(y / \gamma_{r}+z\right) f\left(y / \gamma_{r}+z\right) d y d z \\
\stackrel{(4)}{=} & \left(\prod_{j=1}^{r-1} \gamma_{j}\right) \int_{-\infty}^{\infty} \bar{F}^{-1}(z) G_{r-1}\left(\bar{F}(z) \mid \gamma_{1}-\gamma_{r}+1, \ldots, \gamma_{r-1}-\gamma_{r}+1\right) \\
& \times f(z) \bar{F}^{\gamma_{r}}\left(x / \gamma_{r}+z\right) d z
\end{aligned}
$$




$$
\begin{aligned}
= & \left(\prod_{j=1}^{r-1} \gamma_{j}\right) \int_{-\infty}^{\infty} \bar{F}^{-1}(z) G_{r-1}\left(\bar{F}(z) \mid \gamma_{1}-\gamma_{r}+1, \ldots, \gamma_{r-1}-\gamma_{r}+1\right) f(z) \\
& \times \int_{z}^{\infty} \gamma_{r} \bar{F}^{\gamma_{r}-1}\left(x / \gamma_{r}+t\right) f\left(x / \gamma_{r}+t\right) d t d z \\
= & \left(\prod_{j=1}^{r} \gamma_{j}\right) \int_{-\infty}^{\infty} \bar{F}^{\gamma_{r}-1}\left(x / \gamma_{r}+t\right) f\left(x / \gamma_{r}+t\right) \\
& \times \int_{-\infty}^{t} \bar{F}^{-1}(z) G_{r-1}\left(\bar{F}(z) \mid \gamma_{1}-\gamma_{r}+1, \ldots, \gamma_{r-1}-\gamma_{r}+1\right) f(z) d z d t \\
\stackrel{(5)}{=} & \left(\prod_{j=1}^{r} \gamma_{j}\right) \int_{0}^{\infty} \bar{F}^{\gamma_{r}-1}\left(x / \gamma_{r}+t\right) f\left(x / \gamma_{r}+t\right) \\
& \times G_{r}\left(\bar{F}(t) \mid \gamma_{1}-\gamma_{r}+1, \ldots, \gamma_{r-1}-\gamma_{r}+1,1\right) d t \\
\stackrel{(4)}{=} & \left(\prod_{j=1}^{r} \gamma_{j}\right) \int_{0}^{\infty} \bar{F}^{\gamma_{r}-1}\left(x / \gamma_{r}+t\right) f\left(x / \gamma_{r}+t\right) \bar{F}^{1-\gamma_{r}}(t) G_{r}\left(\bar{F}(t) \mid \gamma_{1}, \ldots, \gamma_{r}\right) d t .
\end{aligned}
$$

For $r=1$ this expression reads (using (3))

$$
\begin{aligned}
\gamma_{1} \int_{0}^{\infty} \bar{F}^{\gamma_{1}-1}\left(x / \gamma_{1}+t\right) f\left(x / \gamma_{1}+t\right) & \bar{F}^{1-\gamma_{1}}(t) \bar{F}^{\gamma_{1}-1}(t) d t \\
& =-\left.\bar{F}^{\gamma_{1}}\left(x / \gamma_{1}+t\right)\right|_{0} ^{\infty}=\bar{F}^{\gamma_{1}}\left(x / \gamma_{1}\right) .
\end{aligned}
$$

Thus, the above expression remains valid for $r=1$, since (via (7))

$$
1-F^{D(1)}(x)=1-F^{\gamma_{1} X(1)}(x)=\bar{F}^{\gamma_{1}}\left(x / \gamma_{1}\right), \quad x \in \mathbb{R} .
$$

On the other hand, we have

$$
\begin{aligned}
1-F^{D(r+1)}(x) \stackrel{(8)}{=} & \left(\prod_{j=1}^{r} \gamma_{j}\right) \int_{0}^{\infty} \bar{F}^{-\gamma_{r+1}}(t) G_{r}\left(\bar{F}(t) \mid \gamma_{1}, \ldots, \gamma_{r}\right) f(t) \\
& \times \bar{F}^{\gamma_{r+1}}\left(x / \gamma_{r+1}+t\right) d t
\end{aligned}
$$

Since $F$ is IFR, the function $\log (1-F)$ is concave; thus, we have

$$
\begin{aligned}
\log \bar{F}\left(\frac{x}{\gamma_{r}}+t\right) & =\log \bar{F}\left(\frac{t\left(\gamma_{r}-\gamma_{r+1}\right)}{\gamma_{r}}+\frac{\gamma_{r+1}}{\gamma_{r}}\left(\frac{x}{\gamma_{r+1}}+t\right)\right) \\
& \geq\left(1-\frac{\gamma_{r+1}}{\gamma_{r}}\right) \log \bar{F}(t)+\frac{\gamma_{r+1}}{\gamma_{r}} \log \bar{F}\left(\frac{x}{\gamma_{r+1}}+t\right),
\end{aligned}
$$

which yields

$$
\bar{F}^{\gamma_{r}}\left(x / \gamma_{r}+t\right) \geq \bar{F}^{\gamma_{r}-\gamma_{r+1}}(t) \bar{F}^{\gamma_{r+1}}\left(x / \gamma_{r+1}+t\right)
$$


Because of the IFR property, $h(t) \leq h\left(x / \gamma_{r}+t\right)$ for all $x, t>0$. Hence,

$$
\begin{aligned}
0= & E D(r+1)-E D(r) \\
= & \left(\prod_{j=1}^{r} \gamma_{j}\right) \int_{0}^{\infty} \int_{0}^{\infty} \bar{F}^{1-\gamma_{r}}(t) G_{r}\left(\bar{F}(t) \mid \gamma_{1}, \ldots, \gamma_{r}\right) \\
& \times\left[\bar{F}^{\gamma_{r}-\gamma_{r+1}}(t) h(t) \bar{F}^{\gamma_{r+1}}\left(x / \gamma_{r+1}+t\right)-\bar{F}^{\gamma_{r}}\left(x / \gamma_{r}+t\right) h\left(x / \gamma_{r}+t\right)\right] d t d x \\
\stackrel{(9)}{\leq} & \left(\prod_{j=1}^{r} \gamma_{j}\right) \int_{0}^{\infty} \int_{0}^{\infty} \bar{F}^{1-\gamma_{r}}(t) G_{r}\left(\bar{F}(t) \mid \gamma_{1}, \ldots, \gamma_{r}\right) \\
& \times\left[\bar{F}^{\gamma_{r}-\gamma_{r+1}}(t) h(t) \bar{F}^{\gamma_{r+1}}\left(x / \gamma_{r+1}+t\right)\right. \\
& \left.-\bar{F}^{\gamma_{r}-\gamma_{r+1}}(t) \bar{F}^{\gamma_{r+1}}\left(x / \gamma_{r+1}+t\right) h\left(x / \gamma_{r}+t\right)\right] d t d x \\
= & \left(\prod_{j=1}^{r} \gamma_{j}\right) \int_{0}^{\infty} \int_{0}^{\infty} \bar{F}^{1-\gamma_{r+1}}(t) \bar{F}^{\gamma_{r+1}}\left(x / \gamma_{r+1}+t\right) \\
& \times G_{r}\left(\bar{F}(t) \mid \gamma_{1}, \ldots, \gamma_{r}\right)\left[h(t)-h\left(x / \gamma_{r}+t\right)\right] d t d x \leq 0 .
\end{aligned}
$$

Thus, $h(t)=h\left(x / \gamma_{r}+t\right)$ for all $x, t>0$, which implies the assertion.

The case of a distribution function $F$ with the DFR property can be handled along the same lines.

REMARK 3.3. (i) In case of progressive type II censored order statistics (cf. Balakrishnan and Aggarwala 2000) the parameters $\gamma_{1}, \ldots, \gamma_{n} \geq 1$ are decreasingly ordered so that the preceding theorem applies.

(ii) For generalized order statistics with the restriction $m_{1}=\ldots=$ $m_{n-1}=m$ (i.e., $\left.\gamma_{j}=k+(n-j)(m+1), 1 \leq j \leq n\right)$ the assumption $\gamma_{1} \geq \ldots \geq \gamma_{n}$ reduces to $m \geq-1$, stated in Kamps and Gather (1997).

(iii) As can be seen from the proof of Theorem 3.2, the ordering of all parameters $\gamma_{1} \geq \ldots \geq \gamma_{n}>0$ is not necessary. In order to prove the characterization we need the existence of an index $r$ with $E D(r)=E D(r+1)$ and $\gamma_{r} \geq \gamma_{r+1}$ (cf. Corollary 3.4).

Corollary 3.4. Let $X(1), \ldots, X(n)$ be generalized order statistics based on $F$ and parameters $\gamma_{1}, \ldots, \gamma_{n}>0, F^{-1}(0+)=0, F(x)<1$ for all $x>0$, and let $F$ have the IFR or DFR property. If there exists $r, 1 \leq r \leq n-1$, such that $E D(r)=E D(r+1)$ and $\gamma_{r} \geq \gamma_{r+1}$ then $F \equiv \operatorname{Exp}(\lambda)$ for some $\lambda>0$.

Acknowledgements. The paper was written during a stimulating stay at the Banach Center in Warsaw. The authors would like to express their sincere thanks to Tomasz Rychlik for organizing the research group meeting. 


\section{References}

M. Ahsanullah (1978), A characterization of the exponential distribution by spacings, Ann. Inst. Statist. Math. 30A, 163-166.

M. Ahsanullah (1981a), On characterizations of the exponential distribution by spacings, Statist. Hefte 22, 316-320.

M. Ahsanullah (1981b), Record values of exponentially distributed random variables, ibid. 22, 121-127.

N. Balakrishnan and R. Aggarwala (2000), Progressive Censoring, Birkhäuser, Boston.

P. J. Boland, M. Shaked and J. G. Shanthikumar (1998), Stochastic ordering of order statistics, in: N. Balakrishan and C. R. Rao (eds.), Handbook of Statistics, Vol. 16, Amsterdam, Elsevier, 89-103.

W. Chan, F. Proschan and J. Sethuraman (1991), Convex-ordering among functions, with applications to reliability and mathematical statistics, in: H. W. Block et al. (eds.), Topics in Statistical Dependence, IMS Lecture Notes - Monograph Series, Hayward, CA, 121-134.

E. Cramer and U. Kamps (2003), Marginal distributions of sequential and generalized order statistics, Metrika, to appear.

E. Cramer, U. Kamps and T. Rychlik (2003), Unimodality of uniform generalized order statistics, with applications to mean bounds, Ann. Inst. Statist. Math., to appear.

L. Gajek and U. Gather (1989), Characterizations of the exponential distribution by failure rate and moment properties of order statistics, in: J. Hüsler and R. D. Reiss (eds.), Extreme Value Theory, Springer, Berlin, 114-124.

U. Gather, U. Kamps and N. Schweitzer (1998), Characterizations of distributions via identically distributed functions of order statistics, in: N. Balakrishnan and C. R. Rao (eds.), Handbook of Statistics, Vol. 16, Elsevier, Amsterdam, 257-290.

M. Iwińska (2001), Properties of record values with random index characterizing exponential distribution, Fasc. Math. 32, 49-54.

U. Kamps (1995), A Concept of Generalized Order Statistics, Teubner, Stuttgart.

U. Kamps (1999), Order statistics, generalized, in: S. Kotz et al. (eds.), Encyclopedia of Statistical Sciences, Update Vol. 3, Wiley, New York, 553-557.

U. Kamps and U. Gather (1997), Characteristic properties of generalized order statistics from exponential distributions, Appl. Math. (Warsaw) 24, 383-391.

S. C. Kochar (1990), Some partial ordering results on record values, Comm. Statist. Theory Methods 19, 299-306.

A. M. Mathai (1993), A Handbook of Generalized Special Functions for Statistical and Physical Sciences, Clarendon Press, Oxford.

M. Shaked and J. G. Shanthikumar (1994), Stochastic Orders and Their Applications, Academic Press, Boston.

Department of Mathematics

University of Oldenburg

26111 Oldenburg, Germany

E-mail: cramer@mathematik.uni-oldenburg.de kamps@mathematik.uni-oldenburg.de
Department of Mathematics

University of Jordan

Amman 11942, Jordan

E-mail: mraqab@accessme.com.jo

Received on 6.6.2002;

revised version on 11.2.2003 